\title{
Réalité et Histoire : le quatrième membre de Flandre
}

\section{Walter Prevenier}

\section{Citer ce document / Cite this document :}

Prevenier Walter. Réalité et Histoire : le quatrième membre de Flandre. In: Revue du Nord, tome 43, n¹69, Janvier-mars 1961. pp. 5-14;

doi : https://doi.org/10.3406/rnord.1961.2438

https://www.persee.fr/doc/rnord_0035-2624_1961_num_43_169_2438

Fichier pdf généré le 07/04/2018 


\section{REALITE ET HISTOIRE}

\section{LE QUATRIEME MEMBRE DE FLANDRE}

Le XIVe siècle a vu s'accomplir l'adjonction au collège représentatif des Trois Villes de Flandre d'un quatrième élément : le Franc de Bruges ${ }^{1}$. Fait curieux et qui intrigue l'historien. Que vint faire ce pays rural dans un collège traditionellement et exclusivement urbain ? ${ }^{2}$.

Une solution simple et logique semblait s'imposer. Le comte, en lutte perpétuelle avec ses bonnes villes de Flandre, aspirant à briser leur hégémonie politique dans les parlements, a introduit de force un Quatrième Membre: le Franc, un district gouverné par un magistrat de chevaliers, sympathisants des comtes, et dont les intérêts différaient essentiellement et profondément de ceux des Trois Villes. A défaut de pouvoir interroger les textes du XIVe siècle sur les intentions profondes et secrètes des princes et des politiciens, bornons-nous à présenter quelques faits de cette époque tellement compliquée, qu'il faut se méfier de conclusions faciles ou trop hâtives.

Des recherches minutieuses dans l'ensemble des documents inédits et des collections de sources ont démontré qu'au grand jamais le Franc de Bruges n'a joué un rôle de complice comtal aux séances des parlements de Flandre du XIV siècle $^{3}$. Au contraire, les princes eux-mêmes viennent témoigner de sa solidarité absolue avec le groupe des villes dans l'opposition commune contre la conduite du pouvoir central ${ }^{4}$. En outre, le collège échevinal du Franc n'était pas exclusivement composé de nobles, et on constate d'ailleurs que ceux-ci n'entretiennent pas des relations privées particulièrement amicales avec le prince.

1. Le Franc est la châtellenie de Bruges, une des plus grandes circonscriptions territoriales, située au nord-ouest du comté de Flandres ; sa frontière est formée par la mer du Nord et l'Escaut de Nieuport à Biervliet, et par la ligne Biervliet, Eeklo, Ursel, au nord de Tielt et Roulers, Dixmude a la mer du Nord.

2. A noter qu'à côté du collège urbain des Membres, d'autres organes représentatifs fonctionnaient sporadiquement en Flandre. En $1127-28$ des ligues nobles s'étaient formées et rivalisaient d'efforts avec les villes, en vue d'influencer l'élection d'un nouveau comte (J. DHONDT, Ordres ou Puissances. L'exemple des Etats de Flandre, Annales, Econ. Soc. Civ., t. V, 1950, pp. 292-295; F. L. Ganshof, Les Origines du concept de la souveraineté nationale en Flandre, Revue d'Histoire du Droit, t. XVIII, 1950, pp. 135-158. Au XIVe siècle, furent convoqués des parlements élargis (plains parlements) où figurait, en dehors des bonnes villes, le groupe important de petites villes et châtellenies du comté (J. DhONDT, Les origines des Etats de Flandre, Anciens Pays et Assemblées d'États, t. I, 1950, pp. 32-44). A l'étranger, l'influence des populations rurales dans les institutions représentatives restait également assez faible; des exemples à noter en Allemagne (A. Luschin von Ebengreuth, Die Anfaenge der Landstaende, Histor. Zeitschrift, t. LXXVIII, 1897, p. 432), en Italie (Gorizia) et en Languedoc (P. S. LeICHT, I Rurali ed i Parlamenti, Assemblee di "Stati ", Rivista di Storia del Diritto Italiano, t. XXIV, 1951, pp. 147-159).

3. W. Prevenier, Het Brugse Vrije en de Leden van Vlaanderen [Le Franc de Bruges et les Membres de Flandre], Annales de la Société d'Émulation de Bruges, t. XCVI, 1959, pp. 5-63, donne l'exposé détaillé de ces recherches. Nous reprenons ici l'essentiel des résultats.

4. Lettre de Philippe le Hardi à son chancelier Jean Canard, evêque d'Arras : qu'il $z$ [= les Quatre Membres] sont comme alliez ensemble et d'une volenté (Archiv. Gén. du Royaume, Bruxelles, Trésor de Flandre, $\left.\right|^{\text {re }}$ série, $n^{\circ} 840$, sine dato $[=24$ juillet 1399]). 
Les premiers indices de la collaboration du Franc avec les Trois Membres remontent à la période 1310-1322 ${ }^{5}$. Aucune opposition entre les deux partis ne se manifeste. A plusieurs reprises, le district rural agit en effet sur la demande des villes, parfois même directement et ouver tement contre la politique comtale ${ }^{6}$.

Après un assez long intervalle, le Quatrième Membre émergea de nouveau, bien que sporadiquement, à partir de $1350^{7}$; sa présence simultanée aux parlements spontanés des villes (dans lesquels le comte n'avait aucune influence et ne pouvait certainement pas introduire un élément contre le gré des organisateurs) et aux parlements convoqués par le prince (où les villes figurent en "invilés", sans aucune autorité) prouve que le Franc ne fut et ne pouvait être un complice politique formel ni du comte, ni des bonnes villes ${ }^{8}$. Son installation définitive et systématique se produit enfin, très probablement au début du régime bourguignon. Nous avons exposé ailleurs ${ }^{9}$ toutes les raisons qui nous permettent de croire qu'elle fut la suite d'une initiative du Franc lui-même, de ses démarches auprès du prince et des villes.

Comment le Franc de Bruges, pays fertile en produits agricoles, porteur d'une industrie rurale de draps timidement naissante, contribuable fort important, pouvait-il mieux poursuivre ses intérêts économiques, fiscaux et politiques, qu'en s'introduisant dans le collège des Membres, constamment en contact avec les comtes (pour discuter les impôts, ou arracher les avantages les plus divers) et avec les princes et les villes de l'étranger (pour conclure des accords et régler des conflits commerciaux). L importance générale de la châtellenie et plus spécialement sa quote-part fort élevée dans les impôts du comté ${ }^{10}$, justifiaient pleinement ses hautes ambitions.

On observe une certaine logique dans le rôle tenu par le Quatrième Membre, en ce sens que celui-ci a toujours et judicieusement servi ses intérêts privés avec un opportunisme exemplaire. Son attitude envers le comte et les bonnes villes fut cependant compliquée par deux autres tendances politiques. La première est le sens avisé qu'avait le Franc de la légalité, ce qui l'a certainement empêché de suivre inconditionnellement les villes dans toutes leurs aventures anarchiques pendant les nombreux troubles et révolutions du XIVe siècle, et incité à se montrer plus réservé

5. Première trace en 1310 ; voir : Th. de Limburg-Stirum, Godex Diplomaticus Flandriae, Coll. Soc. d'Émul. de Bruges, t. II, Bruges, 1889, p. 162; F. FuncK-Brentano, Les origines de la guerre de Cent Ans. Philippe le Bel en Flandre, Paris, 1896, p. 578.

6. Cette attitude agressive apparaît très nettement en 1322, lors de la révolte de la Flandre Maritime à laquelle le Franc a été intensivement mêlé; le 30 septembre 1322, il s'était associé à une coalition politique des grandes villes (Bruges, Gand et peut-être également Ypres) dirigée contre Louis de Nevers (L. GiLliodTs-van SEveren, Inventaire de la ville de Bruges, t. I, Bruges, 1871 , p. $333, n^{\circ} 282 ;$ p. $\left.335, n^{\circ} 285\right)$.

7. Th. De Limburg-Srinum, Cartulaire de Louis de Male, Coll. Soc. d'Émul. de Bruges, t. I, Bruges, 1898, p. 368.

8. Preuve de l'impuissance des princes à limiter l'activité des parlements spontanés des Membres : la défense de ces convocations sans autorisation ducale préalable, d'août 1391 (W. Prevenier, Handelingen van de Leden en van de Staten van Vlaanderen, 1384-1405 [Actes des Membres et des Etats de Flandre, 1384-1405]. Comm. Roy. d'Hist.. 40. Bruxelles, 1958, p. 436 , annexe $n^{\circ} 3$ ) $n^{\circ}$ a pas du tout été observée (voir cette même édition, p. 67 les $n^{\circ 8} 184$ et suivants).

9. W. Prevenier, Het Brugse Vrije, pp. 53-59.

10. Voir les tableaux de répartition des impôts (Transport de Flandre) de 1309 (Тн. DE Limburg-Stirum, Codex Diplom. Flandriae, t. II, pp. 141-3, no 253) et de 1408 (L. Gilliodtsvan Severen, Inv. ville de Bruges, t. IV, Bruges, 1876, pp. 19-27). Le Franc paie un peu moins que Bruges et Gand, mais plus qu'Ypres ; il dépasse de beaucoup toutes les autres châtellenies. 
dans la lutte contre le pouvoir central ${ }^{11}$. En second lieu, on relève une forte rivalité, pour des raisons diverses ${ }^{12}$, entre la ville de Bruges et le Franc (qui n'est autre que sa propre châtellenie). Cette opposition resta plutôt latente au cours du XIVe siècle et au début du XVe, pour se manifester plus ouvertement à partir du deuxième quart du XV'e siècle. Deux fois au moins, en effet, Bruges a tâché, sans résultat en $1437^{13}$, mais avec plein succès en $1477^{14}$, de faire exclure le pays du collège des Membres.

La présentation du Franc de Bruges comme une création politique comtale du XIVe siècle est un mythe historique ${ }^{15}$, dont la naissance, à notre avis, remonte précisément à ces événements de 1477, auxquels nous venons de faire allusion. Commençons, pour illustrer ce point de vue, par un bref aperçu du climat politique de la Flandre à cette époque.

La mort de Charles le Téméraire près de Nancy en janvier 1477 plaçait l'héritière, Marie de Bourgogne, démunie de troupes et de ressources matérielles, devant de graves problèmes ${ }^{16}$. Immédiatement la faiblesse de sa position provoqua dans ses pays, et particulièrement en Flandre, une renaissance du particularisme dans sa vigueur pré-bourguignonne. Les Etats-Généraux, aussitôt convoqués par la duchesse, n'accordèrent la levée de troupes demandée qu'après d'importantes concessions politiques, formulées dans ce qu'on a appelé le Grand Privilège du 11 février $1477^{17}$. Dirigé contre la centralisation du gouvernement de la dynastie, cet acte abolissait notamment le Parlement de Malines, symbole de cette tendance ${ }^{18}$. En même temps, les villes de Flandre profitant de la situation pour restaurer leur autonomie, ruinée par les ducs, se font accorder des privilèges particuliers. Bruges, par exemple, exige et obtient, le 9 avril 1477, le rétablissement de ses traditions protec-

11. Th. De Limburg-Stirum, Codex Dipl. Flandriae, t. II, pp. 351-2.

12. Sur le plan politique : l'existence du Quatrième Membre limitait sensiblement l'influence de Bruges dans son quartier, dont le Franc était l'élément essentiel. La ville et le district étaient en plus de grands concurrents commerciaux et industriels (industrie drapière). Finalement, un problème d'organisation militaire (notamment le ressort militaire des petites villes enclavées dans le Franc) causait une discorde permanente.

13. Archiv. de l'État à Bruges, Fonds du Franc, Cartulaire no 1, fo 34r-36v (11 février 1437); E. Vanden Bussche, Inventaire des archives de l'État à Bruges, t. II, Bruges, 1884 , p. 8.

14. Archiv. de la ville de Bruges, Charte $n^{\circ} 1153$ (9 avril 1477); éd. dans : Versaemelinge van eenige oude wetten ende privilegiën van de stad Brugge, Bruges, 1787, p. 110.

15. De tous temps, les historiens se sont rendus coupables de créations de " mythes". Plusieurs ont été créés par l'école romantique du XIX $\mathrm{X}^{\mathrm{e}}$ siècle. Nous citons à titre d'exemple le portrait par trop coloré que nous peint J. KERVYN DE LETTENHOve (Histoire de Flandre, t. IV, Bruxelles, 1849, pp. 75-86) du duc de Bourgogne Philippe le Hardi, présenté comme le despote fanatique tyrannisant ses sujets flamands d'opinion Urbaniste pendant le grand Schisme d'Occident de la fin du XIVe et du début du XVe siècle (repris par L. Gilliodts-van Severen, Inv. ville de Bruges, t. III, Bruges, 1875, pp. 233-241). Réfutation brillante avec un appareil critique exemplaire par N. ValoIs, La France et le grand Schisme d'Occident, Paris, 1896, t. II, pp. 235 271. Sur l'intérêt des mythes historiques, particulièrement pour l'histoire culturelle, voir J. Romern, De functie van een historische fictie [La fonction d'un mythe historique], dans : Het Onvoltooid Vcrlcden, Cultuurhistorische studies, Amsterdam, 1948, pp. 218-220.

16. Pour l'ensemble des faits politiques entre 1477 et 1493, nous nous référons une fois pour toutes aux excellents exposés de F. W. N. Hugenholtz, Crisis en herstel van het Bourgondisch gezag, 1477-1493 [Crise et restauration de l'autorité bourguignonne, 1477-1493] dans : Algemene Geschiedenis der Nederlanden, t. IV, Utrecht, 1952, pp. 1-26, et de H. PIRENNE, Histoire de Belgique, t. III' ${ }^{3}$, Bruxelles, 1923, pp. 3-56.

17. Ed. dans: A. S. DE BLÉCOURT-N. JaPIKSE, Klein Plakkaatboek van Nederland, Groningen, 1919, pp. 3-7, d'après Verzameling van XXIV origineele charters, privilegien en keuren van de provincie van Vlaenderen, Gand, 1787-8, n० 10.

18. Hugenholtz, o. c., p. 3. 
tionnistes par le système de l'étape et l'interdiction de la draperie rurale autour de la ville, ainsi que la suppression de son rival politique et économique, le Franc, comme Quatrième Membre ${ }^{19}$. Pour obvier à toutes ces difficultés intérieures et extérieures, Marie de Bourgogne se décida à faire appel à l'aide de l'archiduc Maximilien d'Autriche, fils de l'empereur Frédéric III, et lui proposa le mariage, conclu effectivement par procu ration le 21 avril $1477^{20}$, et accepté par les Etats-Généraux ${ }^{21}$. Grâce à la popularité de la duchesse et à la diplomatie de son mari respectant les conquêtes particularistes des villes tout en s'efforçant de renforcer timidement le pouvoir central, un calme relatif revint temporairement dans les Pays-Bas.

Après la mort prématurée de Marie, en mars 1482, Maximilien dut poursuivre seul cette politique en face d'une opposition plus forte. Les Etats-Généraux, sous la poussée particulière des Etats de Flandre et de la ville de Gand, ne lui ont accordé la fonction de régent qu'en échange d'un droit d'intervention dans la politique étrangère, notamment dans le conflit franco-bourguignon. Pour les Etats, il n'y avait de salut que dans une paix durable qu'ils désiraient à tout prix; sous leur impulsion, les négociations avec la France, menèrent à la conclusion de la paix d'Arras du 23 décembre 1482, si désavantageuse pour Maximilien. Cet accord fut la pomme de discorde dans le conflit continuel entre l'archiduc et ses sujets, plus spécialement ceux de Flandre, qui recherchaient même l'appui du roi de France contre leur propre prince. La mort de Louis XI en août 1483 et la circonstance que seule la Flandre, dont les buts trop particut laristes effrayaient les autres régions, continua l'opposition, renforçaiensensiblement la position de Maximilien et l'incitaient à se mesurer militairement avec les Flamands. Les opérations de l'archiduc réussirent brillamment et aboutirent à la paix de Bruges, le 28 juin 1485, qui obligea les Trois Villes à le reconnaître définitivement comme leur régent légitime ${ }^{22}$. Fait symptomatique pour la restauration du pouvoir central : par un acte du 18 octobre 1485, le Franc de Bruges fut rétabli dans ses prérogatives de Quatrième Membre de Flandre ${ }^{23}$.

Dorénavant la stabilisation de son régime dans les Pays-Bas permet à Maximilien d'Autriche de se consacrer plus intensément à ses intérêts de prince d'Habsbourg. Point pour longtemps. Dès 1487, de nouvelles difficultés en Flandre l'obligeaient cependant à y revenir. La révolte éclate d'abord à Gand, ensuite à Bruges et à Ypres ${ }^{24}$. Les Trois Villes une fois

19. L. Gilliodts-van Severen, Coutume des Pays et Comté de Bruges, Quartier de Bruges, Coutumes de la ville de Bruges, t. II, Bruxelles, 1875, p. 81 ; à la séance du 25 avril-7 mai 1477 des États-Généraux a Louvain n"apparaissent en effet pour la Flandre que les Trois Villes : J. Cuvelier, J. Dhondt, R. Doemaerd, Actes des États Généraux des anciens Pays-Bas, t. I, Actes de 1427 à 1477, C. R. H., 4, Bruxelles, 1948, p. 335. Dans une lettre du 17 avril 1477, adressée à Cand, la ville de Bruges déclara que la duchesse a supprimé le Quatrième Membre à leur demande explicite (P. VAN DUYSE-E. DE BUSSChER, Inventaire analytique des chartes et documents appartenant aux archives de la ville de Gand, Gand, 1867, p. 247, $\mathrm{n}^{\circ}$ 709).

20. H. PirenNe, o.c., pp. 22-23.

21. Cuvelier, Dhondt, Doemaerd, o.c., pp. 337-9.

22. Hugenholtz, o.c., p. 15.

23. Arch. de l'État à Bruges, Fonds du Franc, Charte n ${ }^{\circ} 550$; acte du 18 octobre 1845 inséré dans la confirmation par Philippe le Beau en septembre 1501 (VANDEN Bussche, o.c., t. I, Bruges 1881 , p. 203 ; 1D.o.c., t. II, p. 16, n 23). S'il est vrai que les trois Villes ont proposé spontanément au prince le rétablissement, comme l'acte de Maximilien nous veut le faire croire, ils y furent évidemment obligés par la force des circonstances, et il ne faut pas y voir la preuve d'une soudaine sympathie envers le Franc.

24. Hugenholtz, o.c., pp. 18-20. 
de plus entrent en action pour défendre leur particularisme séculaire! Et ceci avec une énergie inouie, ne reculant même pas devant l'emprisonnement à Bruges de l'archiduc en personne, un des épisodes les plus dramatiques de son règne. En mai 1488, après sa libération à la suite de promesses aussitôt annulées par parjure, la lutte avec Gand et quelques autres factions politiques dans les Pays-Bas continua. Ce n'est que le 19 juillet 1492 que cette ville, à bout de forces et ruinée par des dissensions internes, cédait définitivement au prince par le traité de Kadzand ${ }^{25}$. Entretemps, le 19 janvier $1490^{26}$, Maximilien avait déjà renouvelé l'acte de rétablissement du Quatrième Membre d'octobre 1485, perdu pendant les troubles de cette époque excessivement mouvementée ${ }^{2 ?}$.

Dans les milieux dirigeants du Franc de Bruges, ces événements ont évidemment eu de graves répercussions. Les chefs politiques et le magistrat ont vivement protesté contre la liquidation du Quatrième Membre, demandée et orchestrée par la ville de Bruges ${ }^{28}$. Cette dégradation, mal accueillie, poussait le Franc, presque par réaction naturelle, à rechercher l'appui de la duchesse Marguerite, également en difficultés avec ses grandes villes flamandes. Un des plus actifs leaders du Franc fut le juriste Philippe Wielant, bourgmaistre du district en 1479-80, donc pendant la période de l'abolition ${ }^{29}$.

Au début du XVIe siècle, entre 1519 et $1520^{30}$ plusieurs dizaines d'années après les faits, ce même Wielant rédigea ses célèbres Antiquités de Flandre. Dans ce travail de droit public, l'auteur s'était essentiellement proposé de développer ses points de vue politiques et juridiques. Son but n'avait rien de littéraire ou d'historique. Incidemment, Wielant traite des Etats de Flandre, et s'est donc vu placé devant le problème de l'origine du Quatrième Membre. Les actes et les comptes, ne lui donnèrent, pas plus qu'à nous, une réponse positive et explicite. Contraint de faire appel à la raison et à la logique et ne pouvant se baser que sur l'attitude politique du Franc dans les années 1477 à 1485-90, dont il était grandement respon-

25. Id., o.c., p. 25 ; Pirenne, o.c., p. 54.

26. Arch. de l'État à Bruges, Fonds du Franc, Charte no 506 : reintegre, restitue et restably le terroir du Franc en Quatriesme Membre du pays et conté de Flandres. Cette confirmation est une des mesures de rétablissement du régime d'avant les troubles, prises par Maximilien le 4 janvier 1490 (Id., n' 504) après la conclusion d'une paix à Tours avec les villes de Gand, Bruges, Ypres et autres ; Maximilien y avait affirmé que a nous seul appartiengne commettre les officiers et renouveller les loix.

27. A. E. Bruges, Fonds du Franc, Charte n 550 : lesquelles lettres [du 18 octobre 1485] de reintegration dessus inserees, ceulx de nostre ville de Bruges et leurs adherens a leur derreniere commocion et rebellion advenue et par eulx commise et perpetree es annees IIII×x VII et IIIIxx VIII, prindrent de fait et de force avec pluseurs chartres, lettres de previlege et autres tiltres appartenans a ceulx du Franc hors de leurs mains et publicquement sur le marché de la dicte ville les deschirerent... Voir également : VANDEN Bussche, o.c., t. I, p. 203.

28. Maximilien d'Autriche, dans son acte du 18 octobre 1485 attribue la responsabilité aux Trois Villes ensemble (au moyen de commotions qui lors survindrent les dictes autres trois membres s'avancerent de fait et de force de hoster et abolir le dit Membre du Franc; A. E. Bruges, Fonds du Franc, Charte no 550).

29. E. 1. Strubbe, Ph. Wielant, dans : Biographie Nationale, t. XXVII, Bruxelles, 1938, Ac. Roy. des Sc., Lett. et B.-A. de Belg., col. 281. Wielant fut bourgmestre des échevins en 1479. 1480 (A. E. Bruges, Fonds du Franc, Registres no 216, fo 9v; F. PrIEM, Documents extr. du dépot des arch. de la Fl. occ. à Bruges, $2^{\mathrm{e}}$ serie, t. VII, Bruges, 1850, p. 162). Du 17 septembre 1480 au 15 septembre 1481 Wielant fut receveur du pays du Franc (A. E. Bruges, Fonds du Franc, Registre no 217, Compte du Franc, fo 1r. ; Vanden Bussche, o.c., t. II, p. 54).

30. V. FRIS, Les Antiquités de Flandre de Ph. Wielant, B.C.R.H., t. LXX (5e série, XI), 1901, p. 393 : Wielant rédigea ses Antiquités entre le 28 juin 1519 et le 2 mars 1520 (date de sa mort). 
sable lui-même en sa qualité de bourgmaistre, Wielant a développé la thèse que le duc Philippe le Hardi, aurait créé le Quatrième Membre dans le but $d$ 'introduire les nombreux chevaliers du Franc dans le collège représentatif de la Flandre ${ }^{31}$. Notre éminent juriste n'a-t-il pas actualisé, sous influence des convictions fort loyalistes et monarchiques du magistrat du Franc de la fin du XVe siècle qui furent également les siennes, certaines situations du début de la période bourguignonne, passé déjà lointain dont on ne discernait plus les traits essentiels tout à fait différents de ceux de la fin du règne de cette dynastie ? On peut le croire : les événements de 1477 furent vraiment trop impressionnants, les indications, fournies par les sources du XIVe siècle, trop vagues pour permettre à ce témoin et protagoniste politique de faire preuve en cet endroit de l'objectivité qu'il recherchait dans l'ensemble de son travail, par ailleurs basé sur un travail d'archives assez honnête ${ }^{32}$.

Avec Wielant, nous nous trouvons donc à l'origine de la théorie classique du Franc. Inconscient ou à dessein (nous croyons plutôt à la seconde hypothèse), Wielant a ainsi fourni la justification de son propre point de vue politique de 1478 .

Le procédé de se référer à des situations antérieures pour servir et soutenir une tendance politique, en altérant de façon plus ou moins évidente la vérité historique, s'est encore reproduit au $\mathrm{XVI}^{\mathrm{e}}$ siècle $^{33}$, et il est d'ailleurs resté en vogue sans relâche jusqu'à l'époque la plus récente ${ }^{34}$.

Après Wielant, la thèse du Franc est devenue vite le bien commun des littérateurs. Le premier à se l'approprier - entre 1550 et $1558^{35}$ fut Jean d'Oudegherst. Bien qu'il eut connu Wielant, sa version en est, dans une certaine mesure du moins, indépendante. Il attribue en effet l'adjonction du Quatrième Membre, non à Philippe le Hardi, mais à Louis de Male ${ }^{36}$, et propose bien plus explicitement comme facteur d'interprétation, les connections intimes et les services réciproques entre

31. Ph. Wielant, Recueil des Antiquités de Flandre, dans : J. J. De Smet, Corpus Chronic. Flandriae, C. R. H., $4^{\circ}$, t. IV, Bruxelles, 1865, p. 241.

32. V. FrIS, Essai d'une analyse des Commentarii sive Annales Rerum Flandr. de J. de Meyere, $1^{\text {re }}$ partie, Gand, 1908, pp. 182-3 ; ID., Les Antiquités de Flandre, pp. 400-406: donne une liste des pièces d'archives identifiées.

33. A. C. De Schrevel, Le traité d'alliance conclu en 1339 entre la Flandre et le Brabant renouvelé en 1578, Annales de la Soc. d'Émul. de Bruges, t. LXV, 1915-22, pp. 51-111; en se référant au traité de 1339 comme précédent, les Gantois de 1578 n'altèrent que partiellement la vérité historique ; il y a évidemment des divergences frappantes entre les deux traités : les intentions étaient tout à fait différentes; en 1578, la Hollande et la Zélande se joignent à l'alliance (Id. o.c., p. 84); c'est à tort qu'on présente en 1578 le traité de 1339 comme un accord entre les États de Brabant et les États de Flandre qui n'existaient pas encore à ce moment-là (Id., o.c., p. 101). Voir également : P. FRÉDERICQ, Le renouvellement en 1578 du traité d'alliance conclu à lépoque de Jacques van Artevelde entre la Flandre et le Brabant, dans: Travaux du Cours prat. d'hist. Nat., $1^{\text {er }}$ fasc., Gand-La Haye, 1883, pp. 123-141.

34. Nous pensons aux événements récents en France, où la création des Comités de Salut Public nous rappelle la fin du XVIII e siècle. En 1955, les Poujadistes avaient proposé dans le programme de leur parti la création d'États-Généraux, projet auquel se rattachaient pour eux des réminescences historiques de 1789 (Keesings Historisch Archief, t. X, $\mathrm{n}^{\circ}$ 1256, pp. 12.308-9).

35. Jean d'Oudegherst a composé en effet son exposé sur l'histoire du Franc probablement pendant qu'il fut pensionnaire du district, c'est-à-dire entre 1551 et 1558 ; il mourut en 1559 (Biographie Nationale, t. XVI, Bruxelles, 1901, col. 385-6).

36. J. D'Oudegherst, Comment et depuis quand le Francq est devenu ung des quatre membres de Flandres, dans : F. Priem, Précis anal. des doc. de la Flandre occid. à Bruges, $2^{\mathrm{e}}$ série, t. IV, Bruges, 1846, p. 258; fait partie d'un recueil de copies d'extraits des comptes du Franc depuis 1397, rédigé par Oudegherst en sa fonction de pensionnaire (texte original : A. E. Bruges, Fonds 
le comte et les nobles loyaux du district au milieu du XIVe siècle ${ }^{37}$. On a donc l'impression que cette version est partiellement l'œuvre personnelle de Jean d'Oudegherst, basée peut-être sur une source inconnue plus vieille encore, existant à côté du texte de Wielant.

En tout cas, cela nous fait supposer qu'au début du XVIe siècle déjà, un mythe dûment établi à propos du Franc s'était répandu comme opinio communis en Flandre, dont se sont inspirés d'une part un Wielant pour des raisons politiques, d'autre part un Jean d'Oudegherst dans un but plus littéraire. Avec ces intentions tout à fait divergentes, tous deux se sont rendus coupables d'un procédé typique : la projection dans le passé des situations et rapports contemporains qui les entourent. Leur récit n'est point basé sur les sources de l'époque ${ }^{38}$. Il relate tout simplement ce que, au XVIe siècle, on pensait des événements du temps de Louis de Male et de Philippe le Hardi, non ce qui s'est vraiment passé alors avec le Franc. Il est clair que nous ne pouvons en tenir compte pour l'interprétation scientifique.

L'œuvre de Jean d'Oudegherst ne fut pas publiée en son temps, et n'a donc probablement pas exercé une influence directe ${ }^{39}$. Mais indirectement bien par son fils Pierre d'Oudegherst, dont les Annales de Flandres parurent en $1571^{40}$. Traditionnellement, on était d'avis que Pierre a simplement refondu le livre de Wielant, en copiant textuellement des passages complets des Antiquités de Flandre ${ }^{41}$; son seul mérite résiderait dans le fait $d$ avoir inséré plusieurs actes authentiques dans son travail ${ }^{42}$. D'autres présentent les choses comme si Pierre n'avait été que l'éditeur des auvres de son père ${ }^{43}$. Ni l'une ni l'autre hypothèse ne semblent exactes, après une confrontation des divers exposés des trois auteurs au sujet de l'affaire du Franc: Pierre d'Oudegherst a puisé pour ses Annales aussi bien dans le manuscrit de son père ${ }^{44}$, que dans l'ouvre de Wielant ${ }^{45}$. Il a même repris scs sources d'information d'unc manic̀rc si peu critique, qu'il n'a pas reculé devant le fait de situer dans un chapitre de son ouvrage, l'admission du Franc sous Louis de Male ${ }^{46}$ - comme son père - et dans un autre chapitre, sous Philippe le Hardi ${ }^{47}$ - comme Wielant laissant au lecteur le choix éventuel.

du Franc, Registres, $n^{\circ}$ 616, fo 30r. et suiv. ; VANDEN Bussche, o.c., t. II, p. 82). Comme Baltyn plus tard, Jean d'Oudegherst avait donc effectivement fouillé les pièces d'archives, qui ne lui ont cependant pas fourni la solution du problème.

37. ID., Ibid., lequel seigneur [ = Louis de Male] connoissant les loyaulx et fructueulx services des dits nobles du Franc, les print à son conseil et les fit appeller [à] toutes convocations signament dimportance notable.

38. Il est intéressant de remarquer que Jean d'Oudegherst cherche partout à appuyer ses affirmations par des références aux sources d'archives, mais qu'il omet soigneusement de le faire pour sa thèse du Franc (Id., o.c., pp. 258-260).

39. Il n'y a que l'édition par F. Priem, citée dans les notes précédentes. L'essentiel de l'œuvre de Jean d'Oudegherst est repris dans les livres de son fils Pierre.

40. P. D'Oudegherst, Annales de Flandres, Anvers, 1571. Curriculum vitæ de l'auteur dans : Biographie Nationale, t. VI, Bruxelles, 1878, col. 145-150.

41. V. FrIs, Les Antiquités, pp. 393-4.

42. V. FrIs, La Cronycke van den lande ende graefscepe van Vlaenderen de N. Despars, B.C. R.H., t. LXX (5e série, XI), 1901 , p. 545 (n. 1).

43. Biographie Nationale, t. XVI, col. 385.

44. Cf. les textes de J. D'OudEGHERst, o.c., p. 258 (voir l'extrait cité plus haut) et de P. D'OUDEGHERST, o.c., pp. 275-6 [... le dict conte Louys print à son conseil lesdicts nobles du Franc les faisant appeller en toutes communications et assemblees...].

45. Cf. P. Wielant, o.c., p. 241, avec P. D'OUdeGherst, o.c., p. 297.

46. P. D'OUdEGHERST, o.c., pp. 275-6.

47. ID., Ibid., p. 297. 
Wielant, la source probablement primaire, est le point de départ de trois chaînes de fliation. A côté de celle de Pierre d'Oudegherst, déterminée accessoirement par la version de son père, une deuxième est composée par le groupe Meyerus - Marchantius - Sanderus. Il ne s'agit pas d'une reprise textuelle, mais plutôt d'une inspiration. Meyerus, en effet, n'attribue pas l'admission du Quatrième Membre au premier duc de Bourgogne, mais à Philippe le Bon et met l'accent sur le conflit entre Bruges et le Franc ${ }^{48}$. Marchantius paraphrase Meyerus, et cite en plus, explicitement l'argument de la prépondérance des nobles dans le banc échevinal du Franc ${ }^{49}$. Sanderus reprend presque mot à mot la version de Marchantius ; c'est donc à tort qu'il cite in margine la variante de Meyerus comme sa source ${ }^{50}$.

Une troisième fliation relie Wielant à Guicciardini, qui donne cependant une interprétation assez divergente. On continue toujours à supposer une initiative princière : le chroniqueur italien hésite notamment entre Philippe le Hardi, comme Wielant l'admet, et Philippe le Bon, comme dans la version Meyerus ${ }^{51}$. Mais c'est surtout dans l'explication de l'adjonction qu'il témoigne d'une vision originale : Guicciardini présente en effet la création du Quatrième Membre comme le résultat de l'accroissement progressif du pouvoir du Franc ${ }^{52}$. Cette thèse est reprise au XVIII ${ }^{e}$ siècle par Pierre Beaucourt de Noortvelde ${ }^{53}$, et se rapproche beaucoup plus de notre propre interprétation ${ }^{54}$, que de la classique.

Plus ou moins indépendant de la théorie classique, le chroniqueur Baltyn a fourni dans son œuvre Beschrijvinge van het Vrije [Description du Franc] de $1604^{55}$, un effort louable pour appuyer ${ }^{56}$ la thèse par des sources d'archives. D'où la date précise de 1393 comme moment d'admission ${ }^{57}$, puisée évidemment dans un acte ou un compte quelconque où le Franc figure à côté des Trois Villes. Pour l'interprétation du rôle du Franc Baltyn reprenait cependant tout simplement le point de vue de Guicciardini.

Grâce aux versions de Wielant et d'une quantité d'autres auteurs, le mythe du Franc est resté populaire pendant toute la période moderne. Et non seulement dans le monde de la littérature et de l'histoire. Son influence s'est fait sentir également dans les milieux politiques : né d'un point de 289.

48. J. Meyerus, Commentarii sive Annales rerum Flandricarum, Anvers, 1561, pp. 288b et

49. J. Marchantius, Flandria commentariorum lib. IIII descripta, etc., Anvers, 1596, pp. $139-140$.

50. A. Sanderus, Flandria illustrata, t. I, Cologne, 1641, p. 281, col. 1.

51. L. Guicciardini, Descrittione di tutti i Paesi Bassi, altrimenti detti Germania inferiore, Anvers, 1567, pp. 229-230.

52. ID., Ibid.: Cosi crescendo giornalmente l'animo con la potenza a Francotti $[=$ habitants du Franc de Bruges].

53. P. Beaucourt De Noortvelde, Jaerbocken van den Vryen, t. I, Bruges, 1785, p. 98 : ... het [Vrije] heeft allengskens ' $t$ voordeel gekregen van andermael mede gedagvaerd te worden met de andere dry Leden van Vlaendere [peu à peu le Franc a obtenu le privilège d'être convoqué à nouveau avec les trois autres Membres de Flandre].

54. Notamment ce point de notre étude sur le Franc, où nous insistons sur l'accroissement de la conscience politique du Franc et de son changement essentiel d'élément passif en défenseur actif de ses propres intérêts entre 1310 et 1386 (W. PrevenIER, Het Brugse Vrije, pp. 53-54).

55. VANDEN BUSSCHE, o.c., t. II, pp. 85-6.

56. Baltyn était greffier du Franc de 1583 a 1624, et a été chargé de la rédaction des Coutumes du Franc de Bruges. Il travaillait, tout comme pour son travail historique, à partir de sources authentiques (Biographie Nationale, t. 1, Bruxelles, 1866, col. 680-2).

57. A. Baltyn, Nauwkeurige beschrijvinge van het land van den Vryen, in : F. PrIEM, o.c., t. IX, pp. 7-8; texte original : A. E. Bruges, Fonds du Franc, Registres, $n^{\circ} 630$, fo $3 r$ en v. 
de vue politique dans le dernier quart du XVe siècle, le mythe fut déterré de nouveau au XVIII ${ }^{e}$ siècle par le gouvernement central, et en particulier par le ministre plénipotentiaire de l'impératrice autrichienne, Cobenzl, comme argument tactique contre les principaux, c'est-à-dire les Membres et le Clergé, en opposition avec les éléments de second degré, appelés subalternes, afin de justifier l'ingérence du pouvoir central dans les Etats de Flandre en 1754 .

A l'époque contemporaine, enfin, il n'a rien perdu de sa force. A partir de considérations certainement plus scientifiques, mais s'appuyant néanmoins toujours sur un préjugé déjà séculaire, il fut débité de nouveau au XIXe siècle par l'infatigable éditeur et archiviste brugeois Gilliodtsvan Severen ${ }^{59}$, par A. Gallet-Miry ${ }^{60}$ suivi dans cette voie par le professeur Vanderkindere dans son Siècle des Artevelde ${ }^{61}$. La version formulée par le professeur Henri Pirenne est finalement devenue classique et acceptée comme opinio communis eruditorum ${ }^{62}$. Des dizaines de fois, elle fut reprise dans toutes les publications ressortissant du domaine de l'histoire politique et institutionnelle de la Flandre et de la Belgique au bas moyen âge ${ }^{63}$, et jusque dans les plus récents articles ${ }^{64}$. En s'infiltrant dans l'ensemble des manuels historiques d'enseignement secondaire, il est théoriquement supposé être connu par toute notre jeunesse scolaire.

Concluons. Le mythe du Quatrième Membre de Flandres, arme politique créée par les comtes du XIVe siècle, est né dans l'esprit du juriste Philippe Wielant (1510-1520), sous l'impression des événements de 1477 1490. En 1477, il n'avait cependant pas encore servi comme justification del'ingérence du prince dans l'organisation du régime représentatif, comme il le fut bien au XVIII siècle. D'annotation plus ou moins accidentelle d'un auteur de droit public, la théorie du Franc a donc évolué et s'est mué en cliché littéraire (du XVIe au XVIIIe siècle) et en argument politique, pour devenir à la fin un mythe fort répandu dans les cuvres érudites et popularisantes de la science de l'histoire ${ }^{65}$.

\section{W. Prevenier,}

\section{Assistant à l'Université de Gand.}

58. P. LENDERS, De politieke crisis in Vlaanderen omstreeks het midden der achttiende eeuw. Bijdragen tot de geschiedenis der Aufklaerung in België [La crise politique en Flandre vers le milieu du dix-huitième siècle. Contributions à l'histoire de l'Aufklaerung en Belgique], Verhand. der Kon. VI. Acad. voor Wet., Lett. en Sch. Kunsten v. Belg., Kl. der Lett., nº 25, Brussel, 1956, p. 83.

59. L. Gilliodts-van Severen, Inventaire de Bruges, t. IV, Bruges, 1876, pp. 295, 301-2 et 315 ; cite J. d'Oudegherst, J.-P. Zaman (pour les dates) et des pièces d'archives.

60. A. Gallet-Miry, Les États de Flandre sous les périodes espagnole et autrichienne, Gand, 1892, pp. 12-15; cite Gilliodts-van Severen et J.-P. Zaman (pour la date de l'introduction du Franc).

61. L. Vanderkindere, Le siècle des Artevelde, Bruxelles, 1907², pp. $229-30$; cite Gilliodtsvan Severen.

62. H. Pirenne, Histoire de Belgique, t. $\mathrm{II}^{3}$, Bruxelles, 1922, pp. 202-3 ; cite Gilliodts-van Severen.

63. Nous citons parmi les plus récentes : Dhondt, Ordres ou Puissances, p. 298 ; Id., Les origines des Etats de Flandre, pp. 44-45 ; J. Gilissen, Le régime représentatif avant 1790 en Belgique, Coll. Notre Passé, Bruxelles, 1952, p. 54 ; F. L. Ganshof, La Flandre, [dans F. Lot-R. Fawtier, Histoire des institutions francaises au moyen âge, $t$. $I$, Institutions Seigneuriales, Paris, 1957], p. 377.

64. H. Konnigsberger, The States General of the Netherlands before the Revolt, in : Studies presented to the Intern. Comm. for the hist. of represent. and parliament. instit., $X^{t b}$ Intern. Congress of Hist. Sc., Rome, 1955, t. XVIII, Louvain-Paris, 1958, p. 145.

65. Nous tenons à remercier vivement le professeur $\mathrm{Eg}_{\mathrm{g}}$. I. Strubbe, de l'Université de Gand, qui ne nous a pas épargné ses conseils judicieux et ses encouragements réconfortants 
pour cette note. Nous rappelons avec plaisir et reconnaissance qu'une question de notre ami le Dr R. Van Caenegem, chargé de cours à l'Université de Gand, posée après notre exposé sur le problème du Franc au Congrès flamand des Philologues (voir : Handelingen van het $X X I I^{\mathrm{e}}$ Vlaams Filologencongres, Leuven, 1957, pp. 307-311) nous a particulièrement incité à entreprendre cette courte étude. Nous exprimons enfin toute notre gratitude envers notre ancien professeur et ami, le Dr M. Van Houtryve, qui a bien voulu revoir notre texte.

\section{ANNEXE}

\section{TABLEAU DU MYTHE DU FRANC}

En guise d'illustration de notre texte nous avons jugé intéressant de présenter un petit tableau des filiations. Il ne prétend nullement être complet.

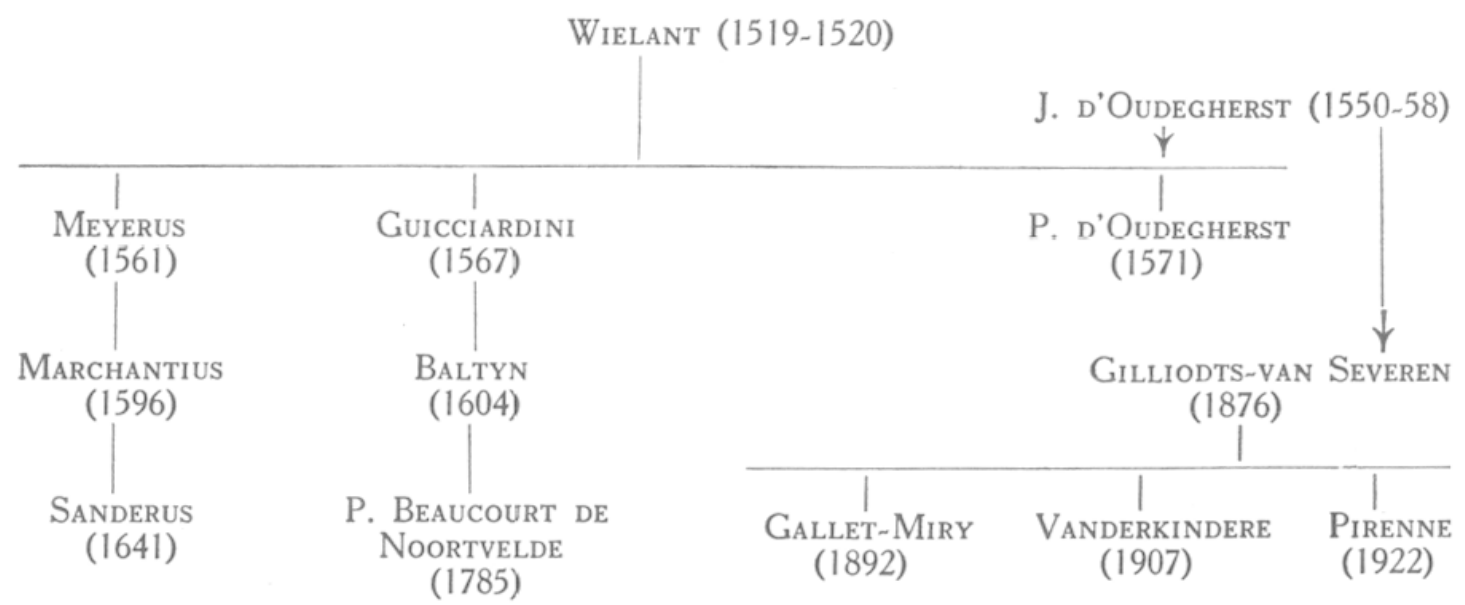

